\title{
Role of Metastasis Suppressor KAI1/CD82 in Different Cancers
}

\author{
Wei Yan $\mathbb{D}^{1,2,3}$ Jinny Huang $\mathbb{D},{ }^{1}$ Qian Zhang $\mathbb{D}^{3}$, and Jian Zhang $\mathbb{D}^{3}$ \\ ${ }^{1}$ Department of Surgery, Johns Hopkins University School of Medicine, Baltimore, MD, USA \\ ${ }^{2}$ Hepatobiliary Center of The First Affiliated Hospital, Nanjing Medical University and Research Unit of Liver Transplantation \\ and Transplant Immunology, Chinese Academy of Medical Sciences, Nanjing, China \\ ${ }^{3}$ Department of General Surgery, The First Affiliated Hospital of Nanjing Medical University, Nanjing, China
}

Correspondence should be addressed to Qian Zhang; zqsummer1031@163.com and Jian Zhang; dr_jianzhang@njmu.edu.cn

Received 19 March 2021; Revised 30 June 2021; Accepted 1 July 2021; Published 9 July 2021

Academic Editor: Nihal Ahmad

Copyright ( $\odot 2021$ Wei Yan et al. This is an open access article distributed under the Creative Commons Attribution License, which permits unrestricted use, distribution, and reproduction in any medium, provided the original work is properly cited.

\begin{abstract}
Metastasis is one of the characteristics of malignant tumors and the main cause of death worldwide. The process of metastasis is mainly affected by tumor metastasis genes, tumor metastasis suppressor genes, tumor microenvironment, extracellular matrix degradation, and other factors. Thus, it is essential to elucidate the mechanism of metastasis and find the therapeutic targets in order to prevent the development of malignant tumors. KAI1/CD82, a member of tetraspanin superfamily of glycoproteins, has been reported as a tumor metastasis suppressor gene in various types of cancers without affecting the tumor formation. Many studies have demonstrated that low expression of KAI1/CD82 might lead to poor prognosis due to its interactions with other tetraspanins and integrins, resulting in the regulation of cell motility and invasion, cell-cell adhesion, and apoptosis. Considering its pathological and physiological significance, KAI1/CD82 could be a potential strategy for clinical predicting and preventing tumor progression and metastasis. The present review aims to discuss the role of KAI1/CD82 in metastasis for different cancers and examine its prospects as a metastasis biomarker and a therapeutic target.
\end{abstract}

\section{Introduction}

Metastasis has been the leading cause of cancer-related mortality. It is well known that tumor metastasis refers to the process in which malignant tumor cells leave the primary site, pass through vessels and then enter blood circulation and lymphatic circulation of the host, and eventually form malignant tumor, the same type of tumor as the primary one $[1,2]$. Metastasis can be affected by a variety of molecular components [3]. Among them, tumor metastasis suppressor genes are a class of specific proteins which can negatively regulate tumor metastasis without affecting the growth of the primary tumor [4]. So far, over 20 metastasis suppressor genes have been discovered, and these genes are always found to be downregulated in different types of cancers $[4,5]$. Therefore, targeting these suppressors is a promising therapeutic strategy for clinically inhibiting tumor metastasis.

Tetraspanins, a highly conserved protein family, are expressed on intracellular membranes and cell surface. 34 tetraspanins are known in mammals, of which 33 are expressed especially in humans [6]. Tetraspanins could affect multiple cell processes including motility, antigen presentation, and receptor-mediated signaling [7]. KAI1, also known as CD82, consists of four transmembrane domains and is a metastasis suppressor gene belonging to the tetraspanin superfamily located on chromosome $11 \mathrm{p} 11.2[8,9]$.

A large number of experiments have shown that the differentially expressed KAI1/CD82 is closely related to malignant tumors, which can be served as a biomarker in tumors [10]. KAI1/CD82 gene was initially cloned as a human gene that could inhibit the metastatic ability of rat AT6.1 prostate cancer cells. In a mouse xenograft model, it was shown that enforced expression of KAI1/CD82 could repress lung metastasis of AT6.1 cells without affecting the growth rate of the primary tumor [11]. KAI1/CD82 expression is decreased in malignant tumors and is closely related to malignant progression, metastasis, and prognosis, containing breast, colon, lung, ovarian, nasopharyngeal, 
liver, and pancreatic cancer [12-14]. In addition, KAI1/ CD82 influences diverse biologic functions, including cell signal transduction, adhesion, migration, motility, protein trafficking, and aggregation [9]. A recent study showed that using peptide mimicking the small extracellular ring domain of KAI1/CD82 could suppress cell metastasis, invasion, and adherence in vitro and inhibit the pulmonary metastasis in vivo [15].

This review will mainly focus on the understanding of the behavior of KAI1/CD82 in different types of cancers and discuss the mechanism and pathways through which it plays a role in tumor metastasis.

\section{Different Cancers}

2.1. Hepatocellular Carcinoma. Despite the clinically therapeutic progress of hepatocellular carcinoma (HCC) such as liver surgical resection, transplantation, or chemotherapy, the survival rate was less than 5\% within five years, mainly due to the high rate of postoperative recurrence and metastasis after surgical resection $[16,17]$.

Firstly, it is well-known that the KAI1/CD82 mRNA and protein expression was significantly reduced in the tumors compared to adjacent nontumor liver tissues obtained from the same patient [18]. Previous research reported that low expression of miR-362-3p can cause high expression of KAI1/CD82, which suppresses migration and invasion of HCC cells [19]. Additionally, except for miR-363-3p, KAI1/ CD82 was also confirmed to be downregulated by miR-197, while anti-miR-197 could suppress HCC migration and invasion by targeting KAI1/CD82 [18].

Moreover, a study found that the synergistic effects of KAI1/CD82 and ganglioside GM3 or GM2/GM3 were able to inhibit HCC cell motility and migration via epidermal growth factor receptor (EGFR) or cMet-activated Pl3K/Akt signaling pathway [20]. In addition, p53 dysfunction and low expression of JunB (a member of the fos/jun family) are simultaneous, while they may play an important role in downregulating the expression of KAI1/CD82 in HCC [21]. Furthermore, KAI1/ CD82 was also demonstrated to suppress HGF-induced migration of hepatoma cells via upregulation of Sprouty2 [22]. A latest study also proposed that HBV may inhibit the expression of KAI1/CD82 through hypermethylation of the promoter in hepatoma cells, leading to the development of HCC [23].

2.2. Gastric Cancer. Gastric cancer (GC) is one of the most common tumors and the third leading cause of cancer death worldwide [24]. Although great improvements and progress such as surgery and adjuvant chemotherapy have been made, the overall five-year survival rate of GC still remains low, largely due to the lack of typical early symptoms, which means most patients have developed advanced-stage tumor at diagnosis, accompanied with distant metastasis [25]. However, little exploration has been made on promising biomarkers that could decrease the risk of recurrence and metastasis [26].

At both the mRNA and protein level, poorly differentiated GC cell lines showed higher expression of miR-362-3p and lower expression of KAI1/CD82 compared to highly differentiated GC cell lines [19]. Downregulation of miR362-3p and upregulation of KAI1/CD82, which may mediate E-cadherin, $\mathrm{N}$-cadherin, and vimentin expression in GC cells, could result in the inhibition of GC migration and invasion [27].

Furthermore, KAI1/CD82, which could also be regulated by miR-197, suppresses EGFR expression and phosphorylation in EGF- and HGF-dependent manner in Hepa1-6 cells. KAI1/ CD82 knockout significantly promoted the invasion of GC cells through activating the EGFR/ERK1/2-MMP7 pathway [28]. These findings all indicated that KAI1/CD82 may be involved in the process of GC occurrence and development.

2.3. Esophageal Squamous Cell Carcinoma. Esophageal squamous cell carcinoma (ESCC) has the seventh highest incidence and the sixth highest cancer-related mortality worldwide [29]. Because the progression of ESCC is characterized by natural aggression and asymptomatic behavior, the diagnosis of ESCC is usually at an advanced stage, with tumor cells invading the adventitia and metastatic cells entering local and distant lymph nodes [30].

Studies found lowly expressed KAI1/CD82 was significantly correlated with the pathological stage, lymph node metastasis, and poor prognosis in ESCC patients. In ESCC cells, overexpression of KAI1/CD82 significantly decreased TGF- $\beta 1 /$ Smad signal pathway, including TGF- $\beta 1$, Smad $2 / 3$, MMP-2, and MMP-9 level, and thus decreased cell proliferation, invasion, and metastasis [31].

Besides, motility-related protein (MRP-1/CD9), which is considered to inhibit cell motility, could be inversely correlated with lymph node metastasis in ESCC through downregulating KAI1/CD82 expression [32].

2.4. Colorectal Carcinoma. Colorectal carcinoma (CRC) consists of the development of cancer from colon or rectum [33]. CRC ranks third among malignancies worldwide and is the second leading cause of cancer-related deaths [34]. The clinical treatment of CRC mainly depends on the stage of the disease progression, molecular analysis, and the patients' health status [35].

Decreased KAI1/CD82 expression is correlated with distant metastasis and TNM stage, while enhanced KAI1/ CD82 expression is also positively correlated with overall survival $[36,37]$.

Additionally, a previous study also proposed that the KAI1/CD82 expression did not alter in vitro colorectal carcinoma cell proliferation, but the apparent increased ability of cell-cell adhesion and cell aggregation was observed in KAI1/CD82 transfected cells [38]. 90K, a tumorassociated glycoprotein, which could interact with KAI1/ CD82, showed antitumor activity in colorectal cancer cells via suppressing Wnt signaling with a novel mechanism of $\beta$-catenin ubiquitination [39].

2.5. Breast Cancer. Breast cancer (BC) is the most frequently diagnosed cancer in women. It has a variety of tumor heterogeneity, which poses a challenge to the traditional 
biomarkers for early detection and prognosis [40]. The diagnosis of breast cancer mainly depends on imaging techniques and histopathological examination, but such diagnosis has obvious defects of hysteresis and invasion.

KAI1/CD82 expression was apparently lower in tumors than in adjacent tissues and benign breast disease tissues. Lacking KAI1 expression might be closely associated with more aggressive form of breast cancer [41]. Besides, KAI1/ CD82 expression in tissues shaded negatively with the level of KAI1/CD82 in exosomes, indicating that the KAI1/CD82 expression was redistributed from tissues to the blood as breast cancer developed and metastasized [42]. Additionally, knockdown of liprin- $\alpha 1$ protein in breast cancer cells MDAMB-231 and Hs578T controls cell edge protrusions during invasion and metastasis and is encoded by PPF1A1, which could lead to the upregulation of KAI1/CD82 [43]. Furthermore, another study identified and characterized a primary nuclear nonpolyadenylated antisense- (as-) lncRNA, named lncRNA SKAI1BC and representing as "suppressor of KAI1 in breast cancer", could suppress KAI1/ CD82 mRNA and protein expression in triple-negative breast cancer cell line MDA-MB-231 [44].

2.6. Lung Cancer. Nowadays, with continuous environment and lifestyle changes, the incidence of lung cancer (LC) has been increasing. It is now the most common cancer worldwide. Despite increasing knowledge in the development and risks of lung cancer, it remains the leading cause of death in both men and women worldwide. Approximately $85 \%$ of lung cancer patients are non-small cell lung cancer (NSCLC) [45].

The study found that the KAI1/CD82 level was significantly lower in lung tissues of NSCLC patients compared to normal lung tissues. KAI1/CD82 level was negatively correlated with tumor size, metastasis, and TNM stage in NSCLC $[46,47]$. There are several pathways by which KAI1/ CD82 could suppress lung cancer metastasis. First, KAI1/ CD82 enhances the expression of miR-203 and downregulates FZD2 expression, thus suppressing metastasis by inhibiting the Wnt signaling pathway in NSCLC cells [45]. Second, KAI1/CD82 can also inhibit the Wnt signaling pathway by weakening EGFR signaling, which may attenuate metastatic colonization [48]. Downregulation of KAI1/ CD82 could be a critical step involved in EGFR overexpression, and EGFR mutations trigger stronger tumorigenic activity [49]. Third, the invasion, progression, and metastasis of NSCLC cells were shown to be positively correlated with E-CAD and related to low CD82 expression. CD82 may stabilize or strengthen E-cad-dependent intercellular adhesion by regulating $\beta$-catenin-mediated signal transduction on NSCLC cells and prevent cancer cells from seceding from the primary tumor site [50]. Fourth, KAI1/ CD82 downregulated the Rac1 expression through the PI3K/ $\mathrm{Akt} / \mathrm{mTOR}$ pathway and reduced the metastatic phenotype of H1299 cells [51]. Lastly, KAI1/CD82 overexpression in the H1299 cells suppresses the tumor invasiveness and metastatic potential of NSCLC cells by inducing MMP9 inactivation via the upregulation of TIMP1 [52].
2.7. Nasopharyngeal Carcinoma. Nasopharyngeal carcinoma (NPC) is an epithelial carcinoma arising from the nasopharyngeal mucosal lining, with significant geographical differences [53]. NPC generally remains undetected, and diagnosis is often delayed until an advanced stage, when distal-organ metastasis is common [54].

Studies found that the KAI1/CD82 gene was highly expressed in low metastatic NPC cell lines and nonneoplastic NPC tissues, while lowly expressed in high metastatic potential NPC cells and NPC tissues [55]. It was demonstrated that compared with the control group, tumor growth was not suppressed when mice were injected with KAI1/CD82 ${ }^{+}$ EPCs (endothelial progenitor cells), but the incidence of lung metastasis and the number of metastatic foci on the lung surface were significantly reduced [14].

2.8. Prostate Cancer. Prostate cancer (PC) is the most frequently diagnosed cancer in 105 countries in men and is the most common cause of death from malignant tumours [56]. Most PCs are curable, but metastatic forms are associated with lower survival rate; therefore, imaging is needed to detect and track metastases evolution during treatment [57].

In a decade-long free survival study of biochemical failure, men who used KAI1/CD82 negative CPCs treated by radical prostatectomy had a higher rate of biochemical failure compared with men who used positive PCPs [58]. A present study suggests that KAI1/CD82 functions in suppressing TGF- $\beta 1$ - and Wnt-induced EMT in prostate cancer cells by inhibiting the TGF- $\beta 1 / \mathrm{Smad}$ and Wnt/ $\beta$-catenin pathways, resulting in the development of a motile and invasive mesenchymal phenotype related to the initiation of the metastatic cascade [11]. Another result pointed out that KAI1/CD82 suppressed adhesion signaling pathways through lateral interactions with $\alpha_{3} \beta_{1}$ and $\alpha_{5} \beta_{1}$ integrin, thereby inhibiting EMT adhesion of prostate cancer cells to fibronectin matrix and reducing cell migration and invasion ability [59]. In addition, KAI1/CD82 attenuated the activation of $\beta 1$ integrin and downregulated its outside-in signaling. This results in the reduction of focal adhesion formation and fibronectin expression/secretion, which subsequently interferes with cell adhesion properties and motility in human prostate cancer cells [60]. Furthermore, EWI2/PGRL, an immunoglobulin superfamily member, could synergize KAI1/CD82 to inhibit the migration of prostate cancer cells $[61,62]$.

2.9. Melanoma. Melanoma is a type of skin cancer that originates from melanocytes, the specialized melanin-producing cells that mainly reside in the skin [63]. The incidence and mortality rates of melanoma cancer are increasing due to its great potential to metastasize, and it is the leading cause of skin cancer-related deaths [64].

Studies found that downregulation of KAI1/CD82 mRNA expression in human melanoma cell lines was related to loss of heterozygosity or allelic imbalance [65]. One recently discovered function of KAI1/CD82 is to decrease tumor aggressiveness by suppressing U2AF2-mediated CD44 alternative splicing. Overexpression of CD82 


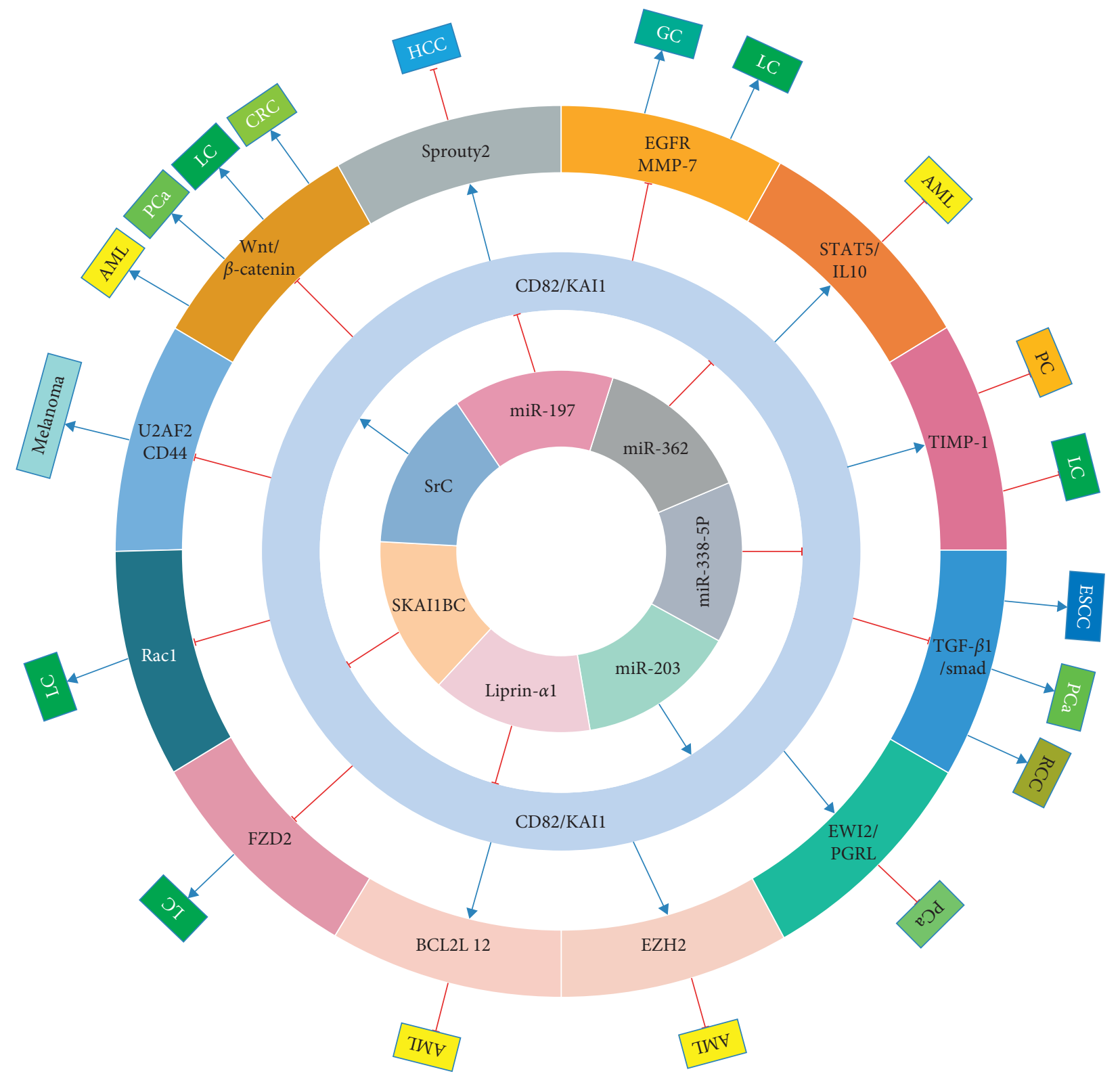

FIgURE 1: Summary of current research on KAI1/CD82 in diverse cancers. HCC: hepatocellular carcinoma; GC: gastric cancer; ESCC: esophageal squamous cell carcinoma; CRC: colorectal carcinoma; BC: breast cancer; LC: lung cancer; NPC: nasopharyngeal carcinoma; PC: prostate cancer; RCC: renal cell carcinoma; AML: acute myelogenous leukemia.

suppressed U2AF2 activity by inducing U2AF2 ubiquitination [66]. This may have potential prognostic and therapeutic implications in melanoma.

KAI1/CD82 mRNA was also identified as a direct target mRNA of miR-338-5p, which is correlated with tumor stage, metastasis, and survival rate [67]. KAI1/CD82 can suppress the ability of melanoma cell invasion and migration by reducing the activity of metalloproteinase- 2 or by using another tumor suppressor protein called inhibitor of growth 4 , which regulates p65 [68]. In highly metastatic melanoma cells, the expression of KAI1/CD82 increased p21 expression upon binding of the Duffy antigen receptor group (DARC). This induces tumor cell senescence and interrupts IL-8mediated vascular endothelial- (VE-) cadherin disassembly
[66]. KAI1/CD82 expression also inhibited melanoma cellinduced gap formation, melanoma cell extravasation in vitro, and subsequent lung metastasis development in vivo [69].

2.10. Acute Myelogenous Leukemia. Acute myelogenous leukemia (AML) originates from hematopoietic stem/progenitor cells and is characterized by the increased numbers of immature myeloid blasts in the bone marrow (BM). AML accounts for approximately $20 \%$ of acute leukemia in children and adolescents [70].

Studies show that KAI1/CD82 inhibits matrix metalloproteinase 9 and augments adhesion of $\mathrm{CD} 34^{+} / \mathrm{CD} 38^{-}$ 
AML cells to the BM microenvironment [71, 72]. KAI1/CD82 can also positively regulate the expression and phosphorylation of EZH2 via inactivation of p38 MAPK signaling in leukemia cells, thus suppressing differentiation programs in leukemic stem cells and augmenting their leukemogenic activity [73]. A recent study revealed a positive correlation between CD82 and BCL2L12 expression (an antiapoptotic protein) at mRNA and protein levels due to STAT5A and AKT signaling in AML cells isolated from patients, which eventually stimulated proliferation and engrafting of leukemia cells [74]. Furthermore, CD82 positively regulated the STAT5/IL-10 signaling pathway in CD $34^{+} / \mathrm{CD}^{-} 8^{-}$AML cells. Downregulation of CD82 dephosphorylated STAT5 which could bind to the promoter region of the IL-10 gene and stimulated IL-10 expression at the transcriptional level, resulting in the decreased level of $\mathrm{IL}^{-10}$ in $\mathrm{CD} 34^{+} / \mathrm{CD} 38^{-}$ AML cells [75]. In addition, in AML children, KAI1/CD82 mRNA expression increases the expression of downstream molecules in the $\mathrm{Wnt} / \beta$-catenin pathway such as $\beta$-catenin, c-myc, cyclinD1, and survivin. These molecules will regulate the proliferation and chemotherapy resistance of AML cells [76]. Another study also shows that an aggressive leukemia phenotype in AML children is related to a mechanism where KAI1/CD82 membrane organization regulates sustained $\mathrm{PKC} \alpha$ signaling [77].

KAI1/CD82 may also serve an important role in the evolution of pediatric acute lymphoblastic leukemia (ALL). The KAI1/CD82 mRNA expression level was significantly higher in the patients with ALL-ND (newly diagnosed), B-cell-ALL, and T-cell-ALL compared with those aged match children without BM disease [78].

\section{Conclusion}

KAI1/CD82 is a well-characterized metastasis suppressor of various solid malignant tumors without affecting primary tumor growth and a recognized biomarker to predict metastatic potential (Figure 1). In detail, KAI1/CD82 deficiency is related to aggressive tumor behaviors, such as high drug resistance, low differentiation grade, and high recurrence rate, as well as decreased disease free and overall survival duration. According to the research above, KAI1/ CD82 is an independent prognostic factor that can predict survival for various tumors, suggesting that it can be used as a novel diagnostic and prognostic biomarker.

Although the structure and function of KAI1/CD82 are well understood, no agent targeting KAI1/CD82 expression or its function is currently available to be used in clinical metastasis therapy. Due to its structure as a transmembrane protein, the standard methods for drug delivery cannot be applied; however, several other strategies can be taken into consideration. Endogenous locus could be induced to upregulate the expression of KAI1/CD82 and viral-mediated gene therapy methods may be used to restore its function. Other potential targets for cancer metastasis therapy could include specifically expressed downstream pathways which are regulated by the loss of KAI1/CD82 or some extracellular domains of KAI1/CD82 which may show tumor metastasis function. Future studies are still needed to fully explicate the role of KAI1/CD82 in certain cancers and also address whether KAI1/CD82 downregulation is a cause or consequence of the progression of cancer. In summary, KAI1/ CD82 may be considered a significant prognostic marker in predicting metastatic manifestation and may be a promising and effective candidate for treatment for certain cancers.

\section{Conflicts of Interest}

The authors declare that there are no conflicts of interest.

\section{Authors' Contributions}

All authors read and approved the final manuscript.

\section{References}

[1] Z. Zhang, J. Li, Y. Ou et al., "CDK4/6 inhibition blocks cancer metastasis through a USP51-ZEB1-dependent deubiquitination mechanism," Signal Transduction and Targeted Therapy, vol. 5 , no. 1, p. $25,2020$.

[2] M.-S. Lee, H.-J. Byun, J. Lee, D.-I. Jeoung, Y.-M. Kim, and H. Lee, "Tetraspanin CD82 represses Sp1-mediated Snail expression and the resultant E-cadherin expression interrupts nuclear signaling of $\beta$-catenin by increasing its membrane localization," Cellular Signalling, vol. 52, pp. 83-94, 2018.

[3] T. Brabletz, D. Lyden, P. S. Steeg, and Z. Werb, "Roadblocks to translational advances on metastasis research," Nature Medicine, vol. 19, no. 9, pp. 1104-1109, 2013.

[4] E. C. Kauffman, V. L. Robinson, W. M. Stadler, M. H. Sokoloff, and C. W. Rinker-schaeffer, "Metastasis suppression: the evolving role of metastasis suppressor genes for regulating cancer cell growth at the secondary site," Journal of Urology, vol. 169, no. 3, pp. 1122-1133, 2003.

[5] L. J. Stafford, K. S. Vaidya, and D. R. Welch, "Metastasis suppressors genes in cancer," The International Journal of Biochemistry and Cell Biology, vol. 40, no. 5, pp. 874-891, 2008.

[6] E. Neumann, M. C. Schwarz, R. Hasseli et al., "Tetraspanin CD82 affects migration, attachment and invasion of rheumatoid arthritis synovial fibroblasts," Annals of the Rheumatic Diseases, vol. 77, no. 11, pp. 1619-1626, 2018.

[7] Y. C. Tsai and A. M. Weissman, "Dissecting the diverse functions of the metastasis suppressor CD82/KAI1," FEBS Letters, vol. 585, no. 20, pp. 3166-3173, 2011.

[8] J. Dong, P. Lamb, C. Rinker-Schaeffer et al., "KAI1, a metastasis suppressor gene for prostate cancer on human chromosome 11p11.2," Science, vol. 268, no. 5212, pp. 884-886, 1995.

[9] N. S. Khan, D. P. Lukason, M. Feliu et al., "CD82 controls CpG-dependent TLR9 signaling," The FASEB Journal, vol. 33, no. 11, pp. 12500-12514, 2019.

[10] P. Habibzadeh, B. Honarvar, M. Silawi et al., "Association between rs2303861 polymorphism in CD82 gene and nonalcoholic fatty liver disease: a preliminary case-control study," Croatian Medical Journal, vol. 60, no. 4, pp. 361-368, 2019.

[11] M. S. Lee, J. Lee, Y. M. Kim, and H. Lee, "The metastasis suppressor CD82/KAI1 represses the TGF-beta 1 and Wnt signalings inducing epithelial-to-mesenchymal transition linked to invasiveness of prostate cancer cells," Prostate, vol. 79, pp. 1400-1411, 2019.

[12] T. Kim, Y. Kim, and H. J. Kwon, "Expression of CD9 and CD82 in papillary thyroid microcarcinoma and its prognostic 
significance," Endokrynologia Polska, vol. 70, no. 3, pp. 224-231, 2019.

[13] J. M. Tam, J. L. Reedy, D. P. Lukason et al., "Tetraspanin CD82 organizes dectin-1 into signaling domains to mediate cellular responses to Candida albicans," The Journal of Immunology, vol. 202, no. 11, pp. 3256-3266, 2019.

[14] G. Wang, L. Zhang, Y. Zhou et al., "KAI1/CD82 genetically engineered endothelial progenitor cells inhibit metastasis of human nasopharyngeal carcinoma in a mouse model," Medical Science Monitor, vol. 24, pp. 3146-3152, 2018.

[15] X. He, X. Ma, C. Wang et al., "The peptide mimicking small extracellular ring domain of CD82 inhibits tumor cell migration in vitro and metastasis in vivo," Journal of Cancer Research and Clinical Oncology, vol. 147, no. 7, pp. 1927-1934, 2021.

[16] Y. Wei, Z. Wang, Y. Zong, D. Deng, P. Chen, and J. Lu, "LncRNA MFI2-AS1 promotes HCC progression and metastasis by acting as a competing endogenous RNA of miR134 to upregulate FOXM1 expression," Biomedicine and Pharmacotherapy, vol. 125, Article ID 109890, 2020.

[17] Y. Li, H. Zuo, H. Wang, and A. Hu, "Decrease of MLK4 prevents hepatocellular carcinoma (HCC) through reducing metastasis and inducing apoptosis regulated by ROS/MAPKs signaling," Biomedicine and Pharmacotherapy, vol. 116, Article ID 108749, 2019.

[18] W. Dai, C. Wang, F. Wang et al., "Anti-miR-197 inhibits migration in HCC cells by targeting KAI1/CD82," Biochemical and Biophysical Research Communications, vol. 446, no. 2, pp. 541-548, 2014.

[19] Q. Zhang, F. Huang, Y. Yao et al., "Interaction of transforming growth factor- $\beta$-Smads/microRNA-362-3p/CD82 mediated by M2 macrophages promotes the process of epithelial-mesenchymal transition in hepatocellular carcinoma cells," Cancer Science, vol. 110, no. 8, pp. 2507-2519, 2019.

[20] Y. Li, X. Huang, J. Zhang, Y. Li, and K. Ma, "Synergistic inhibition of cell migration by tetraspanin CD82 and gangliosides occurs via the EGFR or cMet-activated Pl3K/Akt signalling pathway," The International Journal of Biochemistry and Cell Biology, vol. 45, no. 11, pp. 2349-2358, 2013.

[21] C. Guo, Q. G. Liu, L. Zhang et al., "Expression and clinical significance of p53, JunB and KAI1/CD82 in human hepatocellular carcinoma," Hepatobiliary and Pancreatic Diseases International: HBPD INT.vol. 8, pp. 389-396, 2009.

[22] Z. Mu, H. Wang, J. Zhang, Q. Li, L. Wang, and X. Guo, "KAI1/ CD82 suppresses hepatocyte growth factor-induced migration of hepatoma cells via upregulation of Sprouty2," Science in China Series C: Life Sciences, vol. 51, no. 7, pp. 648-654, 2008.

[23] G. Yu, Y. Bing, W. Li, L. Xia, and Z. Liu, "Hepatitis B virus inhibits the expression of CD82 through hypermethylation of its promoter in hepatoma cells," Molecular Medicine Reports, vol. 10, no. 5, pp. 2580-2586, 2014.

[24] Y. Wu, N. Hao, S. Wang et al., "Long noncoding RNA lncTLN2-4:1 suppresses gastric cancer metastasis and is associated with patient survival," Journal of Oncology, vol. 2020, Article ID 8681361, 2020.

[25] J. Zhang, L. Xing, H. Xu et al., "miR-204-5p suppress lymph node metastasis via regulating CXCL12 and CXCR4 in gastric cancer," Journal of Cancer, vol. 11, no. 11, pp. 3199-3206, 2020.

[26] H. Shen, L. Wang, Q. Chen et al., "The prognostic value of COL3A1/FBN1/COL5A2/SPARC-mir-29a-3p-H19 associated ceRNA network in Gastric Cancer through bioinformatic exploration," Journal of Cancer, vol. 11, no. 17, pp. 4933-4946, 2020.
[27] Q.-H. Zhang, Y.-L. Yao, X.-Y. Wu et al., “Anti-miR-362-3p inhibits migration and invasion of human gastric cancer cells by its target CD82," Digestive Diseases and Sciences, vol. 60, no. 7, pp. 1967-1976, 2015.

[28] L. Xu, Y. Hou, G. Tu et al., "Nuclear Drosha enhances cell invasion via an EGFR-ERK1/2-MMP7 signaling pathway induced by dysregulated miRNA-622/197 and their targets LAMC2 and CD82 in gastric cancer," Cell Death and Disease, vol. 8, Article ID e2642, 2017.

[29] D. Suo, Z. Wang, L. Li et al., "HOXC10 upregulation confers resistance to chemoradiotherapy in ESCC tumor cells and predicts poor prognosis," Oncogene, vol. 39, no. 32, pp. 5441-5454, 2020.

[30] M. M. Forghanifard, S. Azaraz, S. Ardalan Khales, D. Morshedi Rad, and M. R. Abbaszadegan, "MAML1 promotes ESCC aggressiveness through upregulation of EMT marker TWIST1," Molecular Biology Reports, vol. 47, no. 4, pp. 2659-2668, 2020.

[31] T. D. Zeng, B. Zheng, W. Zheng et al., "CD82/KAI1 inhibits invasion and metastasis of esophageal squamous cell carcinoma via TGF-beta1," European Review for Medical and Pharmacological Sciences, vol. 22, pp. 5928-5937, 2018.

[32] S. Uchida, Y. Shimada, G. Watanabe et al., "Motility-related protein (MRP-1/CD9) and KAI1/CD82 expression inversely correlate with lymph node metastasis in oesophageal squamous cell carcinoma," British Journal of Cancer, vol. 79, no. 78, pp. 1168-1173, 1999.

[33] L. Biccas Neto, J. Z. Pulido, G. B. Melo et al., "Photodynamic therapy of presumed choroidal metastasis secondary to colorectal carcinoma: literature review," Case Reports in Ophthalmological Medicine, vol. 2020, Article ID 6490535, 2020.

[34] K. Han, F.-W. Wang, C.-H. Cao et al., "CircLONP2 enhances colorectal carcinoma invasion and metastasis through modulating the maturation and exosomal dissemination of microRNA-17," Molecular Cancer, vol. 19, no. 1, p. 60, 2020.

[35] M. Zoetemelk, G. M. Ramzy, M. Rausch, and P Nowak-Sliwinska, "Drug-drug interactions of irinotecan, 5-fluorouracil, folinic acid and oxaliplatin and its activity in colorectal carcinoma treatment," Molecules (Basel, Switzerland), vol. 25, no. 11,2020 .

[36] E. Zhou, N. Hui, M. Shu, B. Wu, and J. Zhou, "Systematic analysis of the p53-related microRNAs in breast cancer revealing their essential roles in the cell cycle," Oncology Letters, vol. 10, no. 6, pp. 3488-3494, 2015.

[37] D. H. Wu, L. Liu, L. H. Chen et al., "Expression of KAI1/CD82 in human colorectal tumor," Academic Journal of the First Medical College of PLA, vol. 23, pp. 714-715, 2003.

[38] L. Liu, D. H. Wu, Z. G. Li et al., "Effects of KAI1/CD82 on biological behavior of human colorectal carcinoma cell line," World Journal of Gastroenterology, vol. 9, no. 6, pp. 1231-1236, 2003.

[39] J. H. Lee, J. A. Bae, J. H. Lee et al., "Glycoprotein 90K, downregulated in advanced colorectal cancer tissues, interacts with CD9/CD82 and suppresses the Wnt/-catenin signal via ISGylation of beta-catenin," Gut, vol. 59, no. 7, pp. 907-917, 2010.

[40] J. Jeschke, L. Van Neste, S. C. Glöckner et al., "Biomarkers for detection and prognosis of breast cancer identified by a functional hypermethylome screen," Epigenetics, vol. 7, no. 7, pp. 701-709, 2012.

[41] R. Singh, M. L. Bhatt, S. P. Singh et al., "Expression levels of tetraspanin KAI1/CD82 in breast cancers in north Indian females," Asian Pacific Journal of Cancer Prevention: APJCP, vol. 17, no. 7, pp. 3431-3436, 2016. 
[42] X. Wang, W. Zhong, J. Bu et al., "Exosomal protein CD82 as a diagnostic biomarker for precision medicine for breast cancer," Molecular Carcinogenesis, vol. 58, no. 5, pp. 674-685, 2019.

[43] H. Pehkonen, M. Lento, P. von Nandelstadh et al., "Liprin- $\alpha 1$ modulates cancer cell signaling by transmembrane protein CD82 in adhesive membrane domains linked to cytoskeleton," Cell Communication and Signaling, vol. 16, no. 1, p. 41, 2018.

[44] R. Aram, I. Dotan, A. Hotz-Wagenblatt, and D. Canaani, "Identification of a novel metastasis inducing lncRNA which suppresses the KAI1/CD82 metastasis suppressor gene and is upregulated in triple-negative breast cancer," Oncotarget, vol. 8, no. 40, pp. 67538-67552, 2017.

[45] M. Mine, K. Yamaguchi, T. Sugiura et al., "miR-203 inhibits frizzled-2 expression via CD82/KAI1 expression in human lung carcinoma cells," PloS One, vol. 10, no. 7, Article ID e0131350, 2015.

[46] Y. Wang, R. Yang, X. Wang et al., "Evaluation of the correlation of vasculogenic mimicry, Notch4, DLL4, and KAI1/ CD82 in the prediction of metastasis and prognosis in nonsmall cell lung cancer," Medicine, vol. 97, no. 52, Article ID e13817, 2018.

[47] M. Higashiyama, K. Kodama, H. Yokouchi et al., "KAI1/CD82 expression in nonsmall cell lung carcinoma is a novel, favorable prognostic factor," Cancer, vol. 83 , no. 3, pp. 466-474, 1998.

[48] V. V. Prabhu and S. N. Devaraj, "KAI1/CD82, metastasis suppressor gene as a therapeutic target for non-small-cell lung carcinoma," Journal of Environmental Pathology, Toxicology and Oncology, vol. 36, no. 3, pp. 269-275, 2017.

[49] C.-H. Yang, H.-C. Chou, Y.-N. Fu et al., "EGFR over-expression in non-small cell lung cancers harboring EGFR mutations is associated with marked down-regulation of CD82," Biochimica et Biophysica Acta (BBA)-Molecular Basis of Disease, vol. 1852, no. 7, pp. 1540-1549, 2015.

[50] W. U. Shiwu, Y. Lan, S. Wenqing, Z. Lei, and T Yisheng, "Expression and clinical significance of CD82/KAI1 and E-cadherin in non-small cell lung cancer," Archives of Iranian Medicine, vol. 15, no. 11, pp. 707-712, 2012.

[51] U.-J. Choi, B.-K. Jee, Y. Lim, and K.-H. Lee, "KAI1/CD82 decreases Racl expression and cell proliferation through PI3K/Akt/mTOR pathway in H1299 lung carcinoma cells," Cell Biochemistry and Function, vol. 27, no. 1, pp. 40-47, 2009.

[52] B. K. Jee, K. M. Park, S. Surendran et al., "KAI1/CD82 suppresses tumor invasion by MMP9 inactivation via TIMP1 up-regulation in the H1299 human lung carcinoma cell line," Biochemical and Biophysical Research Communications, vol. 342, no. 2, pp. 655-661, 2006

[53] Y. Xiao, J. Qing, B. Li et al., "TIM-3 participates in the invasion and metastasis of nasopharyngeal carcinoma via SMAD7/SMAD2/SNAIL1 axis-mediated epithelial-mesenchymal transition," OncoTargets and Therapy, vol. 13, pp. 1993-2006, 2020.

[54] S. D. Luo, W. C. Chen, C. N Wu et al., "Low-dose aspirin use significantly improves the survival of late-stage NPC: a propensity score-matched cohort study in Taiwan," Cancers, vol. 12, no. 6, 2020.

[55] P. Xu, L. Wang, L. Huang et al., "Identification and characterization of microRNAs expressed in human breast cancer chemo-resistant MCF-7/Adr cells by Solexa deep-sequencing technology," Biomedicine and Pharmacotherapy, vol. 75, pp. 173-178, 2015.
[56] Z. Zhu, Y. Wen, C. Xuan et al., "Identifying the key genes and microRNAs in prostate cancer bone metastasis by bioinformatics analysis," FEBS Open Bio, vol. 10, no. 4, pp. 674-688, 2020.

[57] A. Turpin, E. Girard, C. Baillet et al., "Imaging for metastasis in prostate cancer: a review of the literature," Frontiers in Oncology, vol. 10, p. 55, 2020.

[58] N. P. Murray, S. Aedo, C. Fuentealba et al., "10 Year biochemical failure free survival of men with CD82 positive primary circulating prostate cells treated by radical prostatectomy," Asian Pacific Journal of Cancer Prevention: APJCP, vol. 19, pp. 1577-1583, 2018.

[59] J. Lee, H.-J. Byun, M.-S. Lee et al., "The metastasis suppressor CD82/KAI1 inhibits fibronectin adhesion-induced epithelialto-mesenchymal transition in prostate cancer cells by repressing the associated integrin signaling," Oncotarget, vol. 8, no. 1, pp. 1641-1654, 2017.

[60] H.-A. Lee, I. Park, H.-J. Byun, D. Jeoung, Y.-M. Kim, and H. Lee, "Metastasis suppressor KAI1/CD82 attenuates the matrix adhesion of human prostate cancer cells by suppressing fibronectin expression and $\beta 1$ integrin activation," Cellular Physiology and Biochemistry, vol. 27, no. 5, pp. 575-586, 2011.

[61] X. A. Zhang, W. S. Lane, S. Charrin, E. Rubinstein, and L Liu, "EWI2/PGRL associates with the metastasis suppressor KAI1/ CD82 and inhibits the migration of prostate cancer cells," Cancer Research, vol. 63, no. 10, pp. 2665-2674, 2003.

[62] B. Jee, K. Jin, J.-H. Hahn, H.-G. Song, and H. Lee, "Metastasissuppressor KAI1/CD82 induces homotypic aggregation of human prostate cancer cells through Src-dependent pathway," Experimental and Molecular Medicine, vol. 35, no. 1, pp. $30-37,2003$.

[63] M. Kosmopoulou, A. F. Giannopoulou, A. Iliou et al., "Human melanoma-cell metabolic profiling: identification of novel biomarkers indicating metastasis," International Journal of Molecular Sciences, vol. 21, no. 7, 2020.

[64] B. Sánchez-Sendra, E. Serna, L. Navarro et al., "Transcriptomic identification of miR-205 target genes potentially involved in metastasis and survival of cutaneous malignant melanoma," Scientific Reports, vol. 10, no. 1, p. 4771, 2020.

[65] Y. I. Kim, M. K. Shin, J. W. Lee, J. H. Chung, and M. H Lee, "Decreased expression of KAI1/CD82 metastasis suppressor gene is associated with loss of heterozygosity in melanoma cell lines," Oncology Reports, vol. 21, no. 1, pp. 159-164, 2009.

[66] P. Zhang, S. Feng, G. Liu et al., "CD82 suppresses CD44 alternative splicing-dependent melanoma metastasis by mediating U2AF2 ubiquitination and degradation," Oncogene, vol. 35, no. 38, pp. 5056-5069, 2016.

[67] J. Long, J. Luo, and X. Yin, "MiR-338-5p promotes the growth and metastasis of malignant melanoma cells via targeting CD82," Biomedicine and Pharmacotherapy, vol. 102, pp. 1195-1202, 2018.

[68] Y. Tang, Y. Cheng, M. Martinka, C. J. Ong, and G. Li, "Prognostic significance of KAI1/CD82 in human melanoma and its role in cell migration and invasion through the regulation of ING4," Carcinogenesis, vol. 35, no. 1, pp. 86-95, 2014.

[69] P. Khanna, C.-Y. Chung, R. I. Neves, G. P. Robertson, and C. Dong, "CD82/KAI expression prevents IL-8-mediated endothelial gap formation in late-stage melanomas," Oncogene, vol. 33, no. 22, pp. 2898-2908, 2014.

[70] J. E. Rubnitz, "How I treat pediatric acute myeloid leukemia," Blood, vol. 119, no. 25, pp. 5980-5988, 2012. 
[71] C. Nishioka, T. Ikezoe, and A. Yokoyama, "Blockade of CD82 by a monoclonal antibody potentiates anti-leukemia effects of AraC in vivo," Cancer Medicine, vol. 4, no. 9, pp. 1426-1431, 2015.

[72] C. Nishioka, T. Ikezoe, M. Furihata et al., "CD $34^{+} /$ CD38 ${ }^{-}$acute myelogenous leukemia cells aberrantly express CD82 which regulates adhesion and survival of leukemia stem cells," International Journal of Cancer, vol. 132, no. 9, pp. 2006-2019, 2013.

[73] C. Nishioka, T. Ikezoe, J. Yang, and A. Yokoyama, "Tetraspanin family member, CD82, regulates expression of EZH2 via inactivation of p38 MAPK signaling in leukemia cells," PloS One, vol. 10, no. 5, Article ID e0125017, 2015.

[74] C. Nishioka, T. Ikezoe, A. Takeuchi, A. Nobumoto, M. Tsuda, and A. Yokoyama, "The novel function of CD82 and its impact on BCL2L12 via AKT/STAT5 signal pathway in acute myelogenous leukemia cells," Leukemia, vol. 29, no. 12, pp. 2296-2306, 2015.

[75] C. Nishioka, T. Ikezoe, J. Yang et al., "CD82 regulates STAT5/ IL-10 and supports survival of acute myelogenous leukemia cells," International Journal of Cancer, vol. 134, no. 1, pp. 55-64, 2014.

[76] H. Ji, L. Chen, Y. Xing et al., "CD82 supports survival of childhood acute myeloid leukemia cells via activation of Wnt/ $\beta$-catenin signaling pathway," Pediatric Research, vol. 85, no. 7, pp. 1024-1031, 2019.

[77] C. M. Termini, K. A. Lidke, and J. M. Gillette, "Tetraspanin CD82 regulates the spatiotemporal dynamics of $\mathrm{PKC} \alpha$ in acute myeloid leukemia," Scientific Reports, vol. 6, no. 1, Article ID 29859, 2016.

[78] H. Ji, L. Chen, Y Dai et al., "Aberrant expression of CD133 and CD82 in patients with pediatric acute lymphoblastic leukemia and the clinical significance," Oncology Letters, vol. 14, no. 5, pp. 5811-5818, 2017. 